Reprod. Nutr. Dévelop., 1983, 23 (2 A), 309-319.

\title{
Effets de restrictions alimentaires pendant la croissance des truies sur leur maturité sexuelle et leur reproduction ultérieure
}

\author{
M. ETIENNE, Sylvaine CAMOUS, Annick CUVILLIER \\ avec la collaboration technique de J.-C. HULIN et G. CONSEIL
}

Station de Recherches sur l'Elevage des Porcs, I.N.R.A., St-Gilles, 35590 L'Hermitage, France.

Summary. Effect of feed restrictions during growth on puberty and reproductive performance in gilts.

Effects on reproduction of feed restriction during different periods of growth were investigated in 76 Large-White gilts given one of 3 treatments between 79 days of age $(27.9 \mathrm{~kg})$ and puberty. The control gilts (group 1) were fed ad libitum during the experimental period, and those in group 2 received $12 \%$ less feed from $61 \mathrm{~kg}$ of liveweight until puberty. The gilts in group 3 received $33 \%$ less feed before $61 \mathrm{~kg}$ and were then pair-fed with the gilts in group 1. All the gilts were mated at first estrus and received $2.4 \mathrm{~kg}$ of feed per day during pregnancy. They were laparotomized at 38 days after mating and slaughtered at 105 days of pregnancy to measure early and late embryonic mortality. Contrary to group 3 (age at puberty : 256 days), no difference was found in age at puberty between group 1 and 2 gilts (232 and 226 days, respectively). Early undernutrition delayed age at first estrus, even when followed by normal feeding after $61 \mathrm{~kg}$ of liveweight. The gilts in group 2 had less backfat (backfat depth 24 vs $31 \mathrm{~mm}$ ) and weighed less at puberty than the others $(111 \mathrm{~kg} v s 124 \mathrm{~kg})$. Severe undernutrition tented to increase early embryonic mortality $(21.1,18.8$ and $33 \%$ in groups 1,2 and 3 , respectively) but the reverse was observed thereafter so that there was no difference in total embryonic mortality at 105 days of gestation $(32.6,30.6$ and $36.5 \%$ in groups 1,2 and 3 , respectively).

\section{Introduction.}

L'influence de l'alimentation pendant la croissance sur l'apparition de la puberté chez la truie a fait l'objet de nombreuses expériences qui, en raison de la diversité de leurs conditions et des contradictions entre leurs résultats, ne permettent pas de conclure de façon définitive. II semble pourtant que si un rationnement alimentaire ou énergétique provoque le plus souvent une diminution du poids des femelles au jer cestrus, seule une restriction supérieure à $35 \%$ de l'alimentation à volonté retarde leur puberté (Rérat et Duée, 1976). On peut cependant se demander s'il n'existe pas des moments particuliers de la vie 
de I'animal pendant lesquels sa maturation sexuelle est davantage susceptible d'être affectée par une sous-alimentation. Les données existantes ne permettent pas de répondre, la phase prépubertaire étant généralement incluse dans la période de restriction (Aumaitre et Etienne, 1980). On connaît donc mal les conséquences d'un rationnement des femelles limité à la première partie de leur croissance. Une telle restriction alimentaire chez des porcelets allaités ne modifie pas leur âge au 1er cestrus (Nelson et Robison, 1976). D'après Carrez et al. (1977), les femelles sevrées plus précocement tendraient à avoir une puberté plus tardive. Par ailleurs, des études antérieures ont montré qu'une restriction alimentaire sévère (Etienne et Duée, 1973) ou qu'une déficience en lysine (Duée, 1977) pendant la croissance des truies pouvait accroître la mortalité embryonnaire mesurée au $30^{\circ}$ jour de leur première gestation, mais ces effets n'ont pas été retrouvés sur des femelles pouvant aller jusqu'au terme (Duée, 1977). La présente expérience a donc été entreprise afin de déterminer les effets d'un niveau restreint d'alimentation avant ou après $61 \mathrm{~kg}$ de poids vif sur la précocité sexuelle des truies et, les femelles étant saillies à la puberté, sur les performances de reproduction ultérieures.

\section{Matériel et méthodes.}

L'expérience porte sur 76 porcs femelles de race Large White nés pendant les mois de juin et de juillet. Ils sont répartis au poids de $27,9 \pm 0,4 \mathrm{~kg}$ (1) et à un âge moyen de $79 \pm 1$ jours en 3 lots qui sont soumis à des conditions alimentaires différentes. Ils sont maintenus en groupes de 5 par loge, sur sol paillé, en claustration totale à la température de $18^{\circ} \mathrm{C}$, et alimentés collectivement pendant toute la durée de l'étude. Les mêmes régimes dont la composition figure au tableau 1 sont distribués à tous les animaux sous forme granulée. La substitu-

TABLEAU 1

Composition des aliments

\begin{tabular}{|c|c|c|c|}
\hline & Aliment & Croissance & Finition \\
\hline \multicolumn{4}{|l|}{ Constituants (en \%) : } \\
\hline $\begin{array}{l}\text { Maïs moulu .......... } \\
\text { Tourteau de soja } 50 \ldots \\
\text { Mélasse ............. } \\
\text { Mélange minéral }\left({ }^{1}\right) \\
\text { Mélange vitaminique }{ }^{2}\end{array}$ & 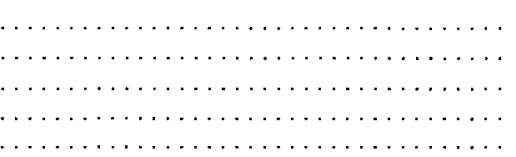 & $\begin{array}{r}71,4 \\
18,6 \\
3,0 \\
3,0 \\
4,0\end{array}$ & $\begin{array}{r}78,5 \\
11,5 \\
3,0 \\
3,0 \\
4,0\end{array}$ \\
\hline
\end{tabular}

(') Mélange minéral apportant $/ \mathrm{kg}$ d'aliment : phosphate bicalcique: $14 \mathrm{~g}$; craie broyée : $10,25 \mathrm{~g}$; sel marin : $5 \mathrm{~g}$; sulfate de cuivre $: 0,04 \mathrm{~g}$; sulfate de zinc $: 0,15 \mathrm{~g}$; sulfate de manganèse : $0,16 \mathrm{~g}$; sulfate de fer: $0,4 \mathrm{~g}$; iodure de potassium : $0,3 \mathrm{mg}$.

(2) Mélange vitaminique apportant/ $\mathrm{kg}$ d'aliment : vitamine $A: 4000 \mathrm{UI}$; vitamine $D_{3}: 400 \mathrm{UI}$; riboflavine: $2 \mathrm{mg}$; pantothénate de calcium : $5 \mathrm{mg}$; acide nicotinique : $10 \mathrm{mg}$; chloline : $40 \mathrm{mg}$; vitamine $\mathrm{B}_{12}: 0,01 \mathrm{mg}$.

(1) Moyenne \pm écart type de la moyenne. 
tion de l'aliment "croissance " par l'aliment "finition " intervient à $61 \mathrm{~kg}$ de poids vif. Les porcs du lot 1 sont nourris à volonté du début de l'expérience à la puberté, tandis que ceux du lot 2, conduits de façon identique jusqu'à $61 \mathrm{~kg}$, sont ensuite rationnés à un niveau de 2,3 $\mathrm{kg} /$ jour. Les femelles du lot 3 reçoivent une quantité restreinte d'aliments qui s'échelonne de $0,8 \mathrm{~kg} / \mathrm{jour}$ avant $30 \mathrm{~kg}$ de poids vif à $2,1 \mathrm{~kg}$ entre 55 et $61 \mathrm{~kg}$. Leur niveau d'alimentation est ensuite égalisé avec la consommation enregistrée sur les animaux du lot 1 lorsqu'ils avaient le même poids. Les changements de régime et de plan d'alimentation sont effectués en fonction de la moyenne du poids des animaux de chaque loge.

Lorsque les jeunes truies atteignent $80 \mathrm{~kg}$ de poids vif, les cestrus éventuels sont détectés par présentation quotidienne à un verrat, et la saillie est effectuée à la première chaleur. Les truies sont alors transférées dans une loge où elles sont alimentées collectivement à raison de $2,4 \mathrm{~kg} /$ jour du régime finition jusqu'à l'abattage effectué à 105 jours de gestation.

Les animaux sont pesés à jeun toutes les deux semaines et leur vitesse de croissance individuelle, la consommation d'aliment et l'indice de consommation moyens par loge sont mesurés et comparés pour chacune des deux périodes : début de l'expérience-61 kg et $61 \mathrm{~kg}$-puberté. L'âge et le poids des femelles à la puberté sont enregistrés.

A $37,9 \pm 0,5$ jours de gestation, les truies n'ayant pas manifesté de retour en cestrus subissent une laparotomie afin de dénombrer corps jaunes et embryons à ce stade. Cette intervention est effectuée sous anesthésie générale obtenue par injection intraveineuse de $1,5 \mathrm{~g}$ de Thiopental sodique et maintenue ensuite par inhalation de $1200 \mathrm{ml} / \mathrm{min}$ d'un mélange gazeux composé de $95 \%$ d'oxygène et de $5 \%$ de fluothane. La paroi abdominale est incisée au niveau de la ligne blanche, et chaque corne utérine est successivement palpée puis réintroduite dans l'abdomen. L'ouverture est ensuite refermée par des sutures à quatre niveaux : péritoine, muscles et aponévroses, couche adipeuse et peau.

Les truies gravides sont abattues à 105, $1 \pm 0,4$ jours de gestation, et le tractus génital est prélevé. Les foetus viables ou morts et les annexes embryonnaires correspondantes sont séparés, dénombrés et pesés. Les ovaires sont pesés, et les corps jaunes disséqués, comptés et pesés. Enfin, le tractus génital est disséqué et mesuré selon une technique décrite par ailleurs (Etienne et Legault, 1974). Les truies revenant en cestrus ou présentant des troubles de la gestation à la suite de la laparotomie sont également abattues afin de mesurer le nombre de corps blancs pour les premières, ou de corps jaunes pour les secondes, de façon à estimer le taux d'ovulation au premier œstrus. De même, les femelles dont la puberté n'a pas été observée avant l'âge de 300 jours sont sacrifiées, et leurs ovaires examinés.

La mortalité embryonnaire est estimée aux deux stades de gestation (38 et 105 jours) par le rapport, exprimé en pourcentage, entre le nombre d'embryons vivants et le taux d'ovulation, lui-même supposé égal au nombre de corps jaunes sur les ovaires. Par la suite, nous distinguerons la mortalité embryonnaire précoce (observée par la laparotomie à 38 jours post-saillie) de la mortalité tardive (calculée par différence entre celles mesurées à l'abattage et à la laparotomie). 
Afin d'apprécier la composition corporelle des truies à 105 jours de gestation, leur carcasse froide est découpée selon la technique parisienne.

Les résultats sont comparés par analyse de la variance et par le test de Newman-Keuls. Les relations éventuelles entre certains paramètres sont recherchées par régression linéaire, curvilinéaire ou polynomiale.

\section{Résultats.}

L'évolution des effectifs d'animaux dans chaque lot entre le début de l'expérience et l'abattage est rapportée dans le tableau 2. Le régime alimentaire n'exerce aucune influence sur les nombres de femelles éliminées soit avant le début des contrôles de chaleurs, soit à l'âge de 300 jours pour causes d'impuberté, de puberté non détectée, ou de non-fécondation. Par ailleurs, une proportion relativement élevée de truies gravides à la laparotomie n'atteint pas 105 jours de gestation $(20 \%)$. Le nombre de femelles dans chaque lot à la fin de l'étude est donc comparable, mais relativement faible par rapport à l'effectif initial.

TABLEAU 2

Devenir des animaux au cours de l'expérience

\begin{tabular}{|c|c|c|c|}
\hline Effectif des femelles & Lot 1 & Lot 2 & Lot 3 \\
\hline Mises en expérience. & 25 & 25 & 26 \\
\hline Eliminées avant $80 \mathrm{~kg} \ldots \ldots$. & 3 & 3 & 3 \\
\hline Impubères à l'âge de 300 jours .. & 2 & 2 & $1\left({ }^{1}\right)$ \\
\hline A puberté non détectée ........ & 2 & 1 & 3 \\
\hline Saillies $\ldots \ldots \ldots \ldots \ldots \ldots$. . . . . & 18 & 19 & 19 \\
\hline 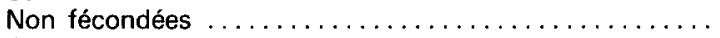 & 4 & 5 & $7\left({ }^{2}\right)$ \\
\hline Gravides à la laparotomie $\ldots \ldots \ldots \ldots \ldots \ldots \ldots$ & 14 & 14 & 12 \\
\hline Abattues à 105 jours de gestation $\ldots \ldots \ldots \ldots \ldots \ldots$ & 10 & 10 & 12 \\
\hline
\end{tabular}

(1) Une truie du lot 3 était en 1re ovulation la veille de son abattage effectué à 309 jours d'âge.

(2) Une truie pubère du lot 3 qui présentait un ovotestis droit, alors que l'ovaire gauche portait 3 corps blancs, a été éliminée après la laparotomie.

1. Performances de croissance et d'engraissement (tabl. 3). - Les performances des animaux avant $61 \mathrm{~kg}$ de poids vif ne font apparaître aucune différence de consommation, de vitesse de croissance ni d'indice de consommation entre les lots 1 et 2 . Le rationnement des femelles du lot 3, correspondant à une diminution de $33 \%$ du niveau d'alimentation par rapport à celles nourries à volonté, provoque une réduction équivalente de la vitesse de croissance sans que l'efficacité alimentaire soit modifiée.

De $61 \mathrm{~kg}$ à la puberté, les performances des truies des lots 1 et 3 sont comparables alors que, conformément au protocole, les femelles du lot 2 ingèrent quotidiennement $12 \%$ d'aliment de moins que les autres, ce qui provoque un ralentissement de la croissance sans affecter l'indice de consommation. 
TABLEAU 3

Performances de croissance et d'engraissement

Lot $\quad \begin{array}{lllll}1 & 2 & 3 & S \bar{x}\left({ }^{1}\right) & \begin{array}{c}\text { Signification } \\ \text { statistique }\left({ }^{2}\right)\end{array}\end{array}$

\begin{tabular}{|c|c|c|c|c|c|}
\hline \multicolumn{6}{|l|}{ Période $28-61 \mathrm{~kg}$ : } \\
\hline $\begin{array}{l}\left.\text { Consommation d'aliment }(\mathrm{kg} / \mathrm{j}) \mathrm{f}^{3}\right) \ldots \ldots \\
\text { Gain moyen quotidien }(\mathrm{g} / \mathrm{j}) \ldots \ldots \ldots \ldots \\
\text { Indice de consommation } \ldots \ldots \ldots \ldots \ldots\end{array}$ & $\begin{array}{l}1,899_{a} \\
617^{a} \\
3,25\end{array}$ & $\begin{array}{r}1,911_{\mathrm{a}} \\
582_{\mathrm{a}} \\
3,36\end{array}$ & $\begin{array}{l}1,27_{\mathrm{b}} \\
393_{\mathrm{b}} \\
3,22\end{array}$ & $\begin{array}{r}0,10 \\
24 \\
0,14\end{array}$ & $\begin{array}{l}* * \\
* * \\
N S\end{array}$ \\
\hline \multicolumn{6}{|l|}{ Période $61 \mathrm{~kg}$-puberté : } \\
\hline $\begin{array}{l}\text { Consommation d'aliment }(\mathrm{kg} / \mathrm{j}) \ldots \ldots \ldots \\
\text { Gain moyen quotidien }(\mathrm{g} / \mathrm{j}) \ldots \ldots \ldots \ldots \ldots \\
\text { Indice de consommation } \ldots \ldots \ldots \ldots \ldots\end{array}$ & $\begin{array}{l}2,54_{a} \\
661_{a} \\
3,82\end{array}$ & $\begin{array}{l}2,24_{b} \\
562 b \\
3,95\end{array}$ & $\begin{array}{l}2,677_{a} \\
707_{a}^{a} \\
3,86^{2}\end{array}$ & $\begin{array}{r}0,09 \\
27 \\
0,21\end{array}$ & NS \\
\hline \multicolumn{6}{|l|}{ Epaisseur de lard $(\mathrm{mm})\left({ }^{4}\right)$ : } \\
\hline $\begin{array}{l}\text { à } 80 \mathrm{~kg} \ldots \ldots \ldots \ldots \ldots \ldots \ldots \ldots \ldots \ldots \ldots \ldots \ldots \ldots \ldots \\
\text { à la puberté }\end{array}$ & $\begin{array}{l}21,3 \\
30,9\end{array}$ & $\begin{array}{l}20,5 \\
23,8_{b}\end{array}$ & $\begin{array}{l}21,4 \\
31,4 a\end{array}$ & $\begin{array}{l}0,7 \\
1,2\end{array}$ & $\begin{array}{l}\text { NS } \\
*^{*}\end{array}$ \\
\hline
\end{tabular}

Pour un même poids vif de $80 \mathrm{~kg}$ correspondant à des âges respectifs de 170, 177 et 192 jours dans les lots 1, 2 et 3, l'épaisseur moyenne du tissu gras sous-cutané est analogue chez tous les animaux. Au contraire, à la puberté, les truies du lot 2 sont plus maigres que les autres.

2. Précocité sexuelle des animaux. - L'âge des femelles au 1er œestrus est comparable dans les lots 1 et 2, tandis qu'il est retardé de près d'un mois chez celles du lot 3 (tabl. 4). II en résulte une consommation globale d'aliment entre le début de l'expérience et la puberté semblable pour les animaux des lots 1 et $3(351 \mathrm{~kg}$ contre $356 \mathrm{~kg})$, alors qu'elle est diminuée de $13 \%$ dans le lot 2 $(306 \mathrm{~kg})$. Le poids de ces truies à la puberté est également significativement plus faible.

3. Performances de reproduction (tabl. 5 et 6 ). - Le taux d'ovulation des femelles du lot 2 rationnées en fin de croissance tend à être plus faible que chez

TABLEAU 4

Caractéristiques des truies à la puberté

\begin{tabular}{|c|c|c|c|c|c|}
\hline Lot & 1 & 2 & 3 & $S \bar{x}$ & $\begin{array}{l}\text { Signification } \\
\text { statistique }\end{array}$ \\
\hline $\begin{array}{l}\text { Age à la puberté }(j) \ldots \\
\text { Poids à la puberté }(\mathrm{kg})\end{array}$ & $\begin{array}{l}232,2 \mathrm{a} \\
124,3 \mathrm{a}\end{array}$ & $\begin{array}{l}225,5_{a} \\
111,0_{b}\end{array}$ & $\begin{array}{l}255,8_{b} \\
123,7_{a}\end{array}$ & $\begin{array}{l}5,7 \\
3,2\end{array}$ & $* * *$ \\
\hline
\end{tabular}


TABLEAU 5

Performances de reproduction

\begin{tabular}{|c|c|c|c|c|c|}
\hline Lot & 1 & 2 & 3 & $S \bar{x}$ & $\begin{array}{l}\text { Signification } \\
\text { statistique }\end{array}$ \\
\hline $\begin{array}{l}\text { Taux d'ovulation } \ldots \ldots \ldots \ldots \ldots \ldots \ldots \\
\text { Nombre d'embryons vivants : }\end{array}$ & 13,6 & 12,0 & 13,7 & 0,5 & $P<0,10$ \\
\hline $\begin{array}{l}\text { - à la laparotomie } \ldots \ldots \ldots \ldots \ldots \ldots \ldots \\
\text { - à } 105 \text { jours de gestation } \ldots \ldots \ldots \ldots \ldots\end{array}$ & $\begin{array}{r}11,4 \\
8,7\end{array}$ & $\begin{array}{r}10,1 \\
8,3\end{array}$ & $\begin{array}{l}9,3 \\
8,3\end{array}$ & $\begin{array}{l}0,4 \\
0,8\end{array}$ & $\begin{array}{l}\text { NS } \\
\text { NS }\end{array}$ \\
\hline Mortalité embryonnaire (p. 100) : & & & & & \\
\hline $\begin{array}{l}\text { - précoce } \ldots \ldots \ldots \ldots \ldots \ldots \ldots \ldots \ldots \ldots \\
- \text { tardive } \ldots \ldots \ldots \ldots \ldots \ldots \ldots \ldots \\
- \text { attribuée à la laparotomie } \ldots \ldots \ldots \ldots \ldots \\
- \text { tardive corrigée }(1) \ldots \ldots \ldots \ldots \ldots \ldots \\
- \text { totale corrigée }(1) \ldots \ldots \ldots \ldots \ldots \ldots\end{array}$ & $\begin{array}{r}21,1 \\
17,7 \\
2,1 \\
15,6_{a} \\
32,6\end{array}$ & $\begin{array}{r}18,8 \\
20,8 \\
6,7 \\
14,1_{a} \\
30,6\end{array}$ & $\begin{array}{r}33,0 \\
6,4 \\
2,9 \\
3,5_{b} \\
36,5\end{array}$ & $\begin{array}{l}5,0 \\
4,5 \\
2,1 \\
3,3 \\
5,5\end{array}$ & $\begin{array}{l}\text { NS } \\
P<0,10 \\
\text { NS } \\
* \\
\text { NS }\end{array}$ \\
\hline
\end{tabular}

(1) Les valeurs corrigées sont obtenues en soustrayant la mortalité attribuée à la laparotomie de la mortalité tardive ou totale mesurées.

TABLEAU 6

Mensurations effectuées sur le tractus génital et les contenus utérins à 105 jours de gestation

\begin{tabular}{|c|c|c|c|c|c|}
\hline Lot & 1 & 2 & 3 & $\mathrm{~S} \bar{x}$ & $\begin{array}{l}\text { Signification } \\
\text { statistique }\end{array}$ \\
\hline Poids des 2 ovaires $(\mathrm{g})$ & 13,7 & 13,9 & 15,3 & 0,8 & NS \\
\hline $\begin{array}{l}\text { Poids moyen des corps } \\
\text { jaunes }(\mathrm{g}) \ldots \ldots \ldots \ldots\end{array}$ & 0,51 & 0,56 & 0,63 & 0,04 & $P<0,10$ \\
\hline $\begin{array}{l}\text { Longueur des } 2 \text { cornes } \\
\text { utérines }(\mathrm{cm}) \ldots \ldots \ldots\end{array}$ & 348 & 339 & 346 & 30 & NS \\
\hline Poids de l'utérus $(\mathrm{kg}) \ldots \ldots \ldots \ldots \ldots$ & 2,81 & 2,73 & 2,85 & 0,17 & NS \\
\hline Poids moyen des fœtus $(g) \ldots \ldots \ldots \ldots$ & 871 & 902 & 976 & 142 & NS \\
\hline Poids des annexes embryonnaires $(\mathrm{g}) \ldots$ & 209 & 229 & 229 & 18 & NS \\
\hline
\end{tabular}

les autres, bien que les écarts ne soient pas significatifs. Le nombre d'embryons vivants n'est affecté par le régime des truies à aucun des deux stades de gestation où il est déterminé. II faut cependant noter que des différences plus importantes à 38 jours post-coïtum que 10 jours avant le terme apparaissent entre les lots. Les observations précédentes mettent en évidence une mortalité embryonnaire précoce non significativement plus élevée dans le lot 3 que dans les autres, alors que la tendance s'inverse par la suite. L'examen de la taille des embryons momifiés trouvés à l'abattage des mères a permis de constater qu'une faible partie d'entre eux sont morts à un stade de gestation proche de la laparotomie sans que cette proportion soit significativement modifiée par les traitements expérimentaux. Si l'on attribue ces décès aux manipulations de l'utérus au cours de l'opération, c'est-à-dire à une cause indépendante des truies et de leurs conditions d'élevage, il est possible de corriger la mortalité tardive qui se trouve alors 
légèrement diminuée. Celle-ci est significativement plus faible chez les femelles du lot 3 sévèrement rationnées jusqu'à $61 \mathrm{~kg}$ alors que la mortalité embryonnaire totale mesurée en fin de gestation et corrigée du nombre des fœetus perdus au cours de la laparotomie est comparable pour toutes les truies.

Les résultats des mensurations effectuées sur le tractus génital gravide et ses contenus sont présentés au tableau 6. Ils ne révèlent aucune différence entre le poids des ovaires ou le développement de l'utérus pendant la gestation des animaux des différents lots. Le poids moyen des corps jaunes tend cependant à être plus élevé chez les femelles du lot 3. Le poids des annexes embryonnaires est semblable dans les trois groupes tandis que celui des fœtus est beaucoup plus variable, mais ne diffère pas significativement quel que soit le régime maternel.

4. Composition corporel/e des truies à l'abattage (tabl. 7). - Compte tenu des écarts de poids vif à l'abattage des truies à 105 jours de gestation, les principaux paramètres de leur composition corporelle sont rapportés en pour cent de leur poids de demi-carcasse. Aucune différence significative ne se manifeste entre les lots. Certaines tendances se dégagent cependant : le poids vif, tout comme le poids de carcasse froide, est plus faible pour les animaux du lot 2 rationnés entre $61 \mathrm{~kg}$ et la puberté, mais le rendement en carcasse n'est pas modifié. La proportion de morceaux maigres (jambon + longe) de la carcasse est supérieure chez ces mêmes femelles, contrairement aux morceaux gras (bardière + pannel. De même, le rapport longe/bardière, plus élevé chez ces truies, indique une moindre adiposité.

TABLEAU 7

Composition corporelle des truies à l'abattage

\begin{tabular}{|c|c|c|c|c|}
\hline Lot & 1 & 2 & 3 & $\begin{array}{l}\text { Signification } \\
\text { statistique }\end{array}$ \\
\hline Poids vif à l'abattage $(\mathrm{kg}) \ldots \ldots \ldots \ldots \ldots \ldots$ & 187 & 176 & 187 & NS \\
\hline Poids de carcasse froide $(\mathrm{kg}) \ldots$ & 126,5 & 120,1 & 127,3 & NS \\
\hline Rendement $(\%) \ldots \ldots \ldots \ldots \ldots \ldots \ldots \ldots \ldots$ & 67,7 & 68,4 & 68,2 & NS \\
\hline Jambon + longe $(\%) \ldots \ldots \ldots \ldots \ldots \ldots \ldots$ & 51,14 & 53,08 & 50,96 & NS \\
\hline Bardière + panne $(\%) \ldots \ldots \ldots \ldots \ldots \ldots \ldots$ & 20,63 & 18,20 & 19,96 & NS \\
\hline Longe/bardière $\ldots \ldots \ldots \ldots \ldots \ldots$ & 1,66 & 2,00 & 1,65 & NS \\
\hline
\end{tabular}

\section{Discussion.}

La restriction alimentaire sévère des femelles du lot 3 avant $61 \mathrm{~kg}$ de poids vif provoque un retard de croissance important : elles atteignent ce poids à l'âge de 166 jours au lieu de 139 jours pour celles nourries à volonté. Leur développement corporel est donc retardé lorsqu'on compare les animaux à un âge donné ; par contre, au poids de $80 \mathrm{~kg}$, l'épaisseur de lard est la même pour tous quel que soit le traitement qu'ils ont subi. De façon plus complète, les résultats de 
découpe des truies des lots 1 et 3 , abattues au même poids (mais pas au même âge), indiquent également qu'elles présentent une composition corporelle analogue en dépit de leur passé nutritionnel différent. Ceci démontre l'aptitude élevée des porcs femelles à subir un rationnement important (Desmoulin et Bourdon, 1971). La moindre adiposité des truies du lot 2 à la puberté comme à l'abattage s'explique par le fait qu'elles parviennent à ces stades au même âge que les truies du lot 1 nourries à volonté, mais à un poids inférieur à cause de la restriction alimentaire qui leur a été imposée à partir de $61 \mathrm{~kg}$.

Les résultats de précocité sexuelle des femelles des lots 1 et 2 confirment la majorité des observations montrant que, contrairement au poids qui est diminué, l'âge des jeunes truies à la puberté n'est pas modifié par un rationnement modéré appliqué pendant tout ou partie de leur croissance (Lodge et MacPherson, 1961 ; O'Bannon et al., 1966 ; Aherne et al., 1976 ; Schilling et Schröder, 1977). L'âge moyen de 229 jours de ces animaux au 1er œstrus est relativement précoce par rapport aux performances moyennes du troupeau dont ils sont issus (247 jours, selon Carrez et al., 1977), mais est plus élevé que les valeurs comprises généralement entre 200 et 220 jours relevées par Legault (1973) sur des truies Large White. Une étude antérieure concluait que dans un tel cas, les écarts constatés entre les âges étaient davantage dus à des conditions de milieu d'élevage différentes qu'au type génétique des porcs (Bolet, Etienne et Legault, 1977).

Une restriction alimentaire précoce (de $33 \%$ par rapport à l'alimentation à volonté) entre 78 et 166 jours d'âge (lot 3) retarde de près d'un mois l'apparition du $1^{\text {er }}$ oestrus bien que les femelles aient été alimentées libéralement au cours des 3 mois le précédant. Ceci démontre qu'un retard de maturité sexuelle peut être observé à la suite d'un rationnement sévère non seulement lorsqu'il est appliqué tardivement (de $45 \mathrm{~kg}$ à la puberté : Haines et al., 1959 ; O'Bannon et al., 1966 ; Holness, 1972 ; Etienne et Duée, 1973 ; Friend, 1976 ; Schilling et Schröder, 1977) mais encore quand il n'est imposé que pendant une phase précoce de la croissance. On constate d'ailleurs dans la présente expérience une corrélation linéaire significative $(r=-0,56 ; P<0,001)$ entre la vitesse de croissance des animaux de 29 à $61 \mathrm{~kg}$ et leur âge au premier œestrus. Des relations analogues ont été établies entre le gain moyen quotidien des femelles de $20 \mathrm{~kg}$ au 1 er œstrus et l'âge à la puberté (Robertson et al., 1951 ; Haines et al., 1959 ; Zimmerman et al., 1960 ; Carrez et al., 1977). L'influence de la vitesse de croissance des femelles pendant leur jeune âge sur leur maturation sexuelle n'est cependant pas surprenante puisque comme dans d'autres espèces, la mise en place des mécanismes physiologiques impliqués dans la cyclicité des chaleurs chez la jeune truie est progressive et débute bien avant le $1^{\mathrm{er}}$ cestrus (Pelletier, Carrez-Camous et Thiery, 1981). La comparaison des femelles des lots 1 et 2 montre que l'établissement de la fonction de reproduction semble indépendant de la croissance corporelle tant que celle-ci n'est pas ralentie trop fortement, et la puberté apparaît alors à un âge caractéristique de l'animal et de sa race. Au contraire, on constate un parallélisme remarquable entre les développements corporel et sexuel des truies des lots 1 et 3 : les retards de croissance des animaux du lot 3 à $61 \mathrm{~kg}$ ou à $80 \mathrm{~kg}$ et leur retard de puberté sont comparables, de l'ordre d'un mois, et le 
1 er œestrus apparaît lorsque les animaux à croissance ralentie ont atteint un poids et une composition corporelle analogues à ceux des témoins. II semble donc que si le développement corporel est affecté de façon importante laussi bien en début de croissance comme dans le lot 3 qu'à l'approche de la puberté dans $d^{\prime}$ autres expériences), le $1^{\text {er }}$ œestrus survient plus tardivement et son déclenchement semble alors relié au poids et à la composition tissulaire de la femelle. Cette étude ne permet pas de connaître les facteurs responsables de la liaison qui existe entre la croissance corporelle et la maturation sexuelle mais selon Levasseur (1977), des modifications des constantes physiques et chimiques corporelles pourraient altérer les relations entre le système nerveux central et les unités sécrétrices neuro-gonadotropes de l'hypothalamus.

Les effectifs de truies impubères à l'âge de 300 jours ou dont la puberté n'a pas été détectée sont faibles et non influencés par les traitements expérimentaux. De même, le taux de fertilité, de $74,5 \%$ en moyenne, est normal pour des truies nullipares et comparable dans les 3 lots. La plupart des éliminations d'animaux après la laparotomie sont dues à des problèmes postopératoires, hernies ombilicales principalement, rencontrés tout au début de l'expérience. Ceci explique que les truies du lot 3 , pubères plus tardivement que les autres, n'ont pas été affectées.

Par rapport au lot 1, le taux d'ovulation des femelles du lot 2, moins lourdes, tend à être diminué, alors qu'il est inchangé chez les truies du lot 3 plus âgées. Une corrélation linéaire significative existe d'ailleurs entre ce paramètre et le poids des animaux au même stade $(r=+0,31)$. Le taux d'ovulation paraît donc davantage relié au poids qu'à l'âge des femelles, ce qui confirme les observations antérieures (Etienne et Duée, 1973 ; Duée, 1977 ; Duée, Treil et Camous, 1980).

Les traitements expérimentaux sont sans conséquences sur la taille de la portée et la mortalité embryonnaire mesurées à 105 jours de gestation. Ce résultat reste inchangé après correction de ces valeurs pour les fœetus morts au cours de la laparotomie, sans doute à la suite de l'anesthésie de la mère ou de la palpation de l'utérus. Le taux de mortalité fœtale ainsi obtenu $133,5 \%$ en moyenne) est conforme aux valeurs généralement rapportées chez la truie (Scofield, 1971). Mais son évolution au cours de la gestation diffère entre les trois lots. Bien que dans tous les cas la mortalité embryonnaire précoce soit responsable de la majeure partie des pertes, elle est accrue chez les femelles dont la maturité a été retardée, comme l'avaient constaté précédemment Etienne et Duée (1973). L'inverse se produit cependant par la suite : les pertes tardives sont élevées chez les truies dont ka puberté est apparue à un âge normal (lots 1 et 2), alors qu'elles sont limitées chez les autres. Ce phénomène de compensation qui se manifeste entre les deux phases de gestation est confirmé par la corrélation linéaire trouvée entre les mortalités fœtales précoce et tardive $(r=-0,39 ; P<$ $0,05)$. La mort de fœtus entre 60 et 70 jours de gestation a parfois été signalée (Pomeroy, 1960) et expliquée par une surpopulation de l'utérus (Webel et Dziuk, 1974). La présente expérience illustre le rôle régulateur exercé par la mortalité embryonnaire sur la taille de la portée. Les écarts entre les taux d'ovulation ou les effectifs de fœtus des différents lots observés à 38 jours se trouvent ainsi nivelés à l'approche de la parturition. Elle montre également que dans certaines 
conditions, les pertes de fœetus pendant la $2^{\mathrm{e}}$ moitié de la gestation peuvent être élevées.

Ainsi, I'appréciation de la survie embryonnaire à partir du dénombrement des fœetus à l'issue du premier mois de gestation ne paraît pas toujours satisfaisante, en particulier lorsque le taux de mortalité précoce est faible comme c'est le cas des truies des lots 1 et 2 de la présente expérience. II convient donc de préciser les conditions dans lesquelles une telle compensation se manifeste et de s'assurer si l'alimentation pendant la croissance des jeunes truies est susceptible d'influencer leurs performances de reproduction au-delà d'un stade précoce de la gestation.

Reçu en septembre 1982

Accepté en novembre 1982

Remerciements. - Les auteurs tiennent à remercier A. Locatelli pour les avoir initiés à la technique de laparotomie.

\section{Références}

AHERNE F. X., CHRISTOPHERSON R. J., THOMPSON J. R., HARDIN R. T., 1976. Factors affecting the onset of puberty, post-weaning estrus and blood hormone levels of Lacombe gilts. Can. J. anim. Sci., 56, 681-692.

AUMAITRE A., ETIENNE M., 1980. Techniques d'élevage et productivité de la truie entre la puberté et la réforme. Journée nationale du Porc, Assoc. Fr. Méd. vét. porc., 37-51.

BOLET G., ETIENNE M., LEGAULT C., 1977. Effets de I'habitat et de l'origine génétique sur les performances d'engraissement et la précocité sexuelle de jeunes truies de race Large White. Ann. Zootech., 26, 255-271.

CARREZ S., TREIL F., DUÉE P. H., AUMAITRE A., 1977. Influences sur la puberté de la truie de sa période de naissance et de sa durée d'allaitement. Ann. Zootech., 26, 621625.

DESMOULIN B., BOURDON D., 1971. La séparation des porcs mâles castrés et des porcs femelles en alimentation restreinte depuis le sevrage. I - Réponses de croissance et d'efficacité nutritionnelle. Journ. Rech. porcine en France, 3, 73-79.

DUEEE P. H., 1977. Influence d'une restriction azotée, à partir de $25 \mathrm{~kg}$ de poids vif, sur les performances de reproduction de la truie. Journ. Rech. porcine en France, 9, 193-198.

DUÉE P. H., TREIL F., CAMOUS S., 1980. Influence de l'apport protéique durant la croissance et la première gestation sur les performances de reproduction et la composition corporelle chez la truie. Ann. Zootech., 29, 121-136.

ETIENNE M., DUÉE P. H., 1973. Effets respectifs des niveaux alimentaires pendant la croissance et le premier mois de gestation sur les performances de reproduction chez la truie nullipare : résultats préliminaires. Ann. Zootech., 22, 453-462.

ETIENNE M., LEGAULT C., 1974. Influence de la race et du régime alimentaire sur la précocité sexuelle de la truie. Journ. Rech. porcine en France, 6, 57-62.

FRIEND D. W., 1976. Nutritional effects on age at puberty and plasma amino acid level in Yorkshire gilts and on chemical composition, nucleic acid, fatty acid and hydroxyproline contents of the uterus. J. anim. Sci., 43, 404-412.

HAINES C. E., WARNICK A. C., WALLACE H. D., 1959. The effect of two levels of energy intake on reproductive phenomena in Duroc Jersey gilts. J. anim. Sci., 18, 347-354.

HOLNESS D. H., 1972. Aspects of puberty in the indigenous gilt. South. Afr. J. anim. Sci,, 2 85-90.

LEGAULT C., 1973. Déterminisme génétique de la précocité sexuelle, du taux d'ovulation, et du nombre d'embryons chez la truie primipare: héritabilité, effet d'hétérosis. Journ. Rech. porcine en France, 5, 147-154.

LEVASSEUR M. C., 1977. Thoughts on puberty. Initiation of the gonadotropic function. Ann. Biol. anim. Bioch. Biophys., 17, 345-361. 
LODGE G. A., MacPHERSON R. M., 1961. Level of feeding during early life and the subsequent reproductive performance of sows. Anim. Prod., 3, 19-28.

NELSON R. E., ROBISON O. W., 1976. Effects of postnatal maternal environment on reproduction of gilts. J. anim. sci., 43, 71-77.

O'BANNON R. H., WALLACE H. D., WARNICK A. C., COMBS G. E., 1966. Influence of energy intake on reproductive performance of gilts. J. anim. Sci, 25, 706-710.

PELLETIER J., CARREZ-CAMOUS S., THIERY J. C., 1981. Basic neuroendocrine events before puberty in cattle, sheep and pigs. J. Reprod. Fert., Suppl. 30, 91-102.

POMEROY R. W., 1960. Infertility and neonatal mortality in the sow. IV - Further observations and conclusions. J. agric. Sci. (Camb.), 54, 57-66.

RÉRAT A., DUÉE P. H., 1976. Conditions d'élevage et alimentation chez la jeune truie : influence sur la carrière ultérieure de l'animal, 15-24. In La conduite du troupeau de reproducteurs porcins. Etudes et Formation vétérinaires, Ed. Point vétérinaire, Maisons-Alfort.

ROBERTSON G. L., GRUMMER R. H., CASIDA L. E., CHAPMAN A. B., 1951. Age at puberty and related phenomena in outbred Chester White and Poland China gilts. J. anim. Sci., 10, 647-656.

SCHILLING E., SCHRÖDER J., 1977. Natürliche und induzierte Geschlechtsreife bei Jungsauen nach unterschiedlicher Aufzuchtfütterung. Tierzüchter, 29, 53-56.

SCOFIELD A. M., 1971. Embryonic mortality, 367-383. In CÓLE D. J. A., Pig production, Butterworths, London.

WEBEL S. K., DZIUK P. J., 1974. Effect of stage of gestation and uterine space on prenatal survival in the pig. J. anim. Sci., 38, 960-963.

ZIMMERMAN D. R., SPIES H. G., RIGOR E. M., SELF H. L., CASIDA L. E., 1960. Effect of restricted feeding, crossbreeding and season on age at puberty in swine. J. anim. Sci., 19, 687-694. 